\title{
Dead-in-shell positions of near-term ostrich embryos
}

\author{
Z. Brand ${ }^{1 \#}$, S.W.P. Cloete ${ }^{2,3}$, I.A. Malecki ${ }^{4,5}$ \& C.R. Brown ${ }^{6}$ \\ ${ }^{1}$ Directorate Animal Sciences, Oudtshoorn, PO Box 351, Oudtshoorn, 6220, South Africa \\ ${ }^{2}$ Directorate Animal Sciences, Elsenburg, Private Bag X1, Elsenburg, 7607, South Africa \\ ${ }^{3}$ Department of Animal Sciences, University of Stellenbosch, Private Bag X1, Matieland, 7602, South Africa \\ ${ }^{4}$ School of Animal Biology, Faculty of Natural and Agricultural Science, University of Western Australia, Crawley, 6009, \\ Australia \\ ${ }^{5}$ UWA Institute of Agriculture, The University of Western Australia, 35, Stirling Highway, Crawley, WA 6009, Australia \\ ${ }^{6}$ Institute of Science and the Environment, University of Worcester, Henwick Grove, Worcester, WR2 6AJ, England
}

(Received 29 February 2016; Accepted 30 November 2016; First published online 6 December 2016)

\author{
Copyright resides with the authors in terms of the Creative Commons Attribution 4.0 South African License. \\ See: http://creativecommons.org/licenses/by/4.0/za \\ Condition of use: The user may copy, distribute, transmit and adapt the work, but must recognize the authors and the South African \\ Journal of Animal Science.
}

\begin{abstract}
The patterns of embryonic development in ostriches, especially in the last stage of hatching, are still not well understood. This study examined between 3468 and 3484 dead-in-shell (DIS) eggs with chicks that died between day 35 and day 42 of artificial incubation. Most DIS chicks were positioned correctly with their heads towards the air cell $(52.6 \%)$. DIS chicks that positioned their heads near the equator of the egg amounted to $46.5 \%$, while a small percentage $(0.9 \%)$ were positioned with their upper body towards the bottom of the egg. More DIS chicks tended to pip internally near the equator of the egg (37.6\%) than DIS chicks that pipped internally through the membranes into the air cell (34.4\%). Most DIS chicks had their heads turned in the correct position from left to right (54.4\%), though their beaks were mostly positioned towards the air cell $(52.9 \%)$. The highest percentage of DIS chicks had their feet in the upwards position $(52.4 \%)$, while $46 \%$ had their feet across or below the head. The wings of all DIS chicks were positioned next to the body. Results from the study showed that most of the DIS chicks were roughly in the correct position, but were still unable to hatch. This warrants future research to investigate the reasons that prevent correctly positioned chicks from hatching.
\end{abstract}

Keywords: Head position, malposition, pipping position

\#Corresponding author: Zanellb@elsenburg.com

\section{Introduction}

Ostriches are an important commercial species in South Africa. Problems of egg quality such as shell thickness, hatching and embryonic development of these economically important domesticated birds are relatively well understood and described, but there is little information on embryogenesis and hatching problems specifically related to ostriches. Artificially incubated ostrich eggs generally have a lower hatching success rate than most domesticated poultry species, with high chick mortality in the period close to pipping (internal pipping through the membrane and external pipping through egg shell) and hatching. A particularly poor understanding of patterns of embryonic development around this period contributes to the high incidence of embryonic mortality (Deeming et al., 1993). Developing ostrich chicks start to turn themselves in the egg to assume the correct pipping position from day 35 of incubation (Deeming, 1994; 1997). Deeming (1995) reported that the correct pipping position for ostrich embryos is reached by day 42 of incubation, with the neck of the chick lying from left to right, with the right foot next to the beak and the left foot positioned in the nape of the neck.

Brown et al. (1996), Deeming (1997) and Ipek \& Sahan (2004) reported that malpositioning of embryos next to the air cell generally results in more than $55 \%$ of embryonic mortality, with more than $70 \%$ of all DIS cases occurring during the pipping stage. It has been suggested that these losses are caused mainly by ineffective incubation equipment, which results in high relative humidity, overheating and often inadequate hygiene management (Brown et al., 1996; Van Schalkwyk et al., 1996). This observation has been confirmed in studies by Sahan (2003) and Ipek \& Sahan (2004). About $25 \%$ of all fertile eggs that failed to hatch contain chicks in one or more of several malpositions (Byerly \& Olsen, 1936). Successful artificial incubation is also affected by a number of factors, such as female age, season, and storage conditions of eggs prior to setting in the incubator, as well as the type of incubator (Blood et al., 1998; Van Schalkwyk, 1999; Brand et al., 2007; 2008; 2011). A better understanding of the positions in which chicks succumb may contribute to 
deeper perception of the development of the chick in the period immediately before hatching. The aim of this study was thus to investigate the positions of the DIS chick and its body parts to improve the understanding of factors that prevent healthy ostrich chicks from hatching.

\section{Materials and Methods}

Eggs were obtained from the commercial ostrich breeding flock at Oudtshoorn Research Farm in the Klein Karoo region of South Africa in the 2006-2008 breeding seasons. Specifics about the flock, management and feeding program are described by Van Schalkwyk et al. (1996), Bunter \& Cloete (2004) and Brand et al. (2007). Eggs used in this study included three genotypes, namely: South African Blacks, Zimbabwean Blues, Kenyan Red Necks, and various combinations of these breeds.

Between 3472 and 3484 records were used from eggs with DIS embryos 35 days and older. The normal position of the embryo before pipping was used as a reference to identify embryos in malposition (Deeming, 1995). Eggs were collected daily, weighed, and identified by date and paddock (female) of origin. Methods for collection, sanitation and storage at the experimental site were well documented (Van Schalkwyk et al., 1998; 1999; Brand et al., 2007). Details of the incubators and incubation procedures were provided by Cloete et al. (2001) and Brand et al. (2007; 2011).

On day 44 of incubation, eggs that had not hatched were candled to see whether movement could be detected, which would indicate that internal pipping had occurred. These eggs were then opened manually at the air sac area, and the position of the embryo and point of internal pipping, if any, were noted. If internal pipping had taken place, the location was noted. The positions of DIS chicks of all eggs were documented and described, as well as the position of the head, legs, beak and wings. Where appropriate, closely related positions were pooled to ensure that fewer positions were considered, and that positions were represented by as many eggs as possible.

The frequencies of embryos positioned with their heads near the top (near the air cell), equator, and bottom of the eggs were compared with the one-sample Chi²-test (Van Ark, 1990). The frequencies of DIS embryos conforming to specific positions of the head, beak, feet and wing were compared similarly. In these tests, the observed frequencies were assessed against the hypothesis that all the frequencies were even, as described by Van Ark (1990).

\section{Results}

The large number of permutations describing the positions for each of the DIS chicks, its head, feet and beak complicated analysis. The positions for each body part were categorised to ensure adequate numbers for the simplified permutations (Figure 1). Frequencies for the positions of DIS chicks are shown in Table 1. The frequencies of DIS chicks positioned correctly with their heads directed towards the air cell $(52.6 \%)$, and those turned with their heads midway towards the air cell $(46.5 \%)$ were higher $(P<0.05)$ than the minority group, with their heads positioned towards the bottom of the egg $(0.9 \%)$. In $26.8 \%$ of DIS eggs, no signs of internal pipping were observed. Slightly more DIS chicks tended to pip internally at the equator of the egg $(37.6 \%)$ than DIS chicks that pipped internally through the membranes into the air cell $(34.4 \%)$. Only a small percentage of chicks (1.2\%) managed to pip internally at the bottom of the egg.
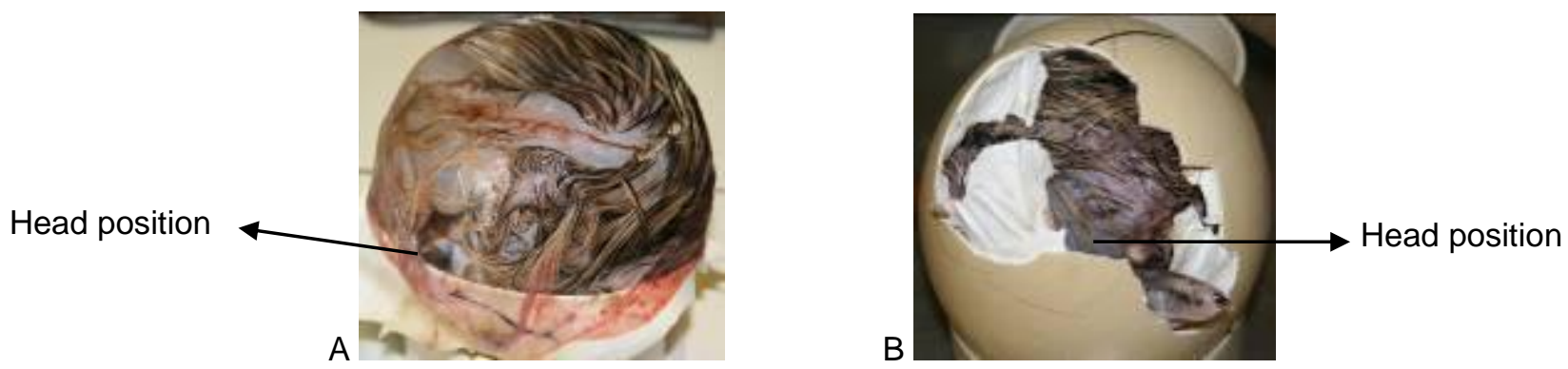

Figure 1 Malposition of dead-in-shell chick with head positioned towards the bottom of the egg (A) and malposition of dead-in-shell chick with head midway towards the air cell (B)

The air cell is denoted with a cross on the egg-shell surface in Figure 1B. 
Table 1 The different positions of dead-in-shell ostrich chicks for the 2006 - 2008 breeding seasons

\begin{tabular}{|c|c|c|c|}
\hline Description & $\begin{array}{c}\text { Number of } \\
\text { records }\end{array}$ & Embryo orientation & $\begin{array}{r}\text { Proportion } \\
(\%) \\
\end{array}$ \\
\hline \multirow[t]{3}{*}{ Chick position } & 3482 & Top by air sac (normal) & 52.6 \\
\hline & & Middle & 46.5 \\
\hline & & Bottom & 0.9 \\
\hline $\operatorname{Chi}^{2}\left(d f^{*}=2\right)$ & & & 1672 \\
\hline \multirow[t]{4}{*}{$\begin{array}{l}\text { Internal pipping } \\
\text { position }\end{array}$} & 3484 & None & 26.8 \\
\hline & & Top by air sac & 34.4 \\
\hline & & Intermediate of egg & 37.6 \\
\hline & & Bottom & 1.2 \\
\hline $\operatorname{Chi}^{2}\left(d f^{\star}=3\right)$ & & & 1139 \\
\hline \multirow[t]{5}{*}{ Head position } & 3474 & Normal (left to right) & 54.4 \\
\hline & & Right to left & 44.6 \\
\hline & & On thigh & 0.2 \\
\hline & & On body & 0.7 \\
\hline & & On wing & 0.1 \\
\hline $\operatorname{Chi}^{2}\left(d f^{\star}=4\right)$ & & & 5130 \\
\hline \multirow[t]{5}{*}{ Beak position } & 3468 & Turned upwards (normal) & 52.9 \\
\hline & & Across or below feet & 1.3 \\
\hline & & Across wing & 44.1 \\
\hline & & Across or below thighs & 1.0 \\
\hline & & Pointed downwards & 0.6 \\
\hline $\operatorname{Chi}^{2}\left(d f^{\star}=4\right)$ & & & 4772 \\
\hline \multirow[t]{4}{*}{ Feet position } & 3484 & Turned upwards (normal) & 52.4 \\
\hline & & Across or below head & 46.0 \\
\hline & & Across or below neck & 1.6 \\
\hline & & Other & 0.1 \\
\hline $\operatorname{Chi}^{2}\left(d f^{\star}=3\right)$ & & & 3286 \\
\hline Wing position & 3472 & Next to body (normal) & 100 \\
\hline
\end{tabular}

The highest percentage of DIS chicks had their heads turned from left to right $(54.4 \%$; considered the correct position) or from right to left (44.6\%). A small number of DIS chicks had their heads in a host of alternate positions, such as on either thigh, on various locations on the body, or turned down and lying on either wing. The most common position for the beak was turned towards the air cell $(52.9 \%$; considered the correct position) and across either wing (44.1\%). Positions that occurred at lower frequencies included the beak lying across or below the feet, across or below either thigh, and pointed downwards.

The highest percentage of DIS chicks had their feet in the upwards position (52.4\%), while $46 \%$ had their feet across or below the head. A small number had their feet across or below the neck or placed elsewhere. The wings were positioned next to the body for all DIS chicks.

\section{Discussion}


A definite pattern was evident for the position of each of the limbs. Most chicks tended to assume the correct pipping position as described by Deeming (1995). Although a high proportion of DIS chicks in the present study were in the correct position, a substantial proportion that pipped internally were positioned with their heads in the equator of the egg. Only a small number $(0.9 \%)$ was presented with their heads towards the bottom of the egg, away from the air cell, and only $1.2 \%$ pipped internally at the bottom of the egg. This finding differs from that of Deeming (1995), who reported that the most common malposition for ostrich embryos is with the head at the opposite end from the air cell. The findings of the current study, however, correspond with those of Ley et al. (1986), who stated that chicks with pipping positions away from the air cell were more likely to succumb to suffocation because they would be unable to penetrate the air cell. If external pipping did occur in these positions, then it is possible that up to $65 \%$ of chicks would survive to hatching (Brand et al., 2011).

The authors' observations on head positions corresponded with the findings by Deeming (1995), who reported that chicks may also be positioned with their heads to the left, instead of to the right, while some get their heads stuck across their right leg, or the right foot becomes stuck over the head or in the beak. Although pipping would still be possible, even though the heads were positioned towards the right, chicks would not be able to pip if the head or beak were obstructed in any way. Results from the current study could be compared with observations by Brown et al. (1996) that common malpositions were legs facing upwards, but with the head facing the opposite pole, with chicks on their sides with their legs in the middle of the egg, with the head turned towards the air cell or towards the bottom of the egg.

The ideal positioning for the feet would be in the upwards position, because they are used in the hatching process to kick against the eggshell (Deeming, 1994). Deeming (1995) also identified the foot positioned on the underside of the head as a problem that prevents some chicks from hatching. The wing position of ostrich chicks differs from that of chickens, where the beak is positioned under the wing at the time of hatching (Deeming, 1994). This study did not attempt to compare malpositions of DIS ostrich chicks with positions in chickens.

Results from the current study showed that approximately $50 \%$ of the DIS chicks were correctly positioned, thus making suffocation because of a malposition unlikely. Contributing factors to the inability of the chicks to hatch could include eggshells that were too thick, making it impossible for chicks to break the shell, low eggshell porosity limiting the oxygen supply, insufficient water loss, causing oedema and excess water loss, causing dehydration (Deeming et al., 1993; Brown et al., 1996).

\section{Conclusion}

It appears that deviations from the normal hatching position vary greatly in ostrich chicks. The current research indicates that about $50 \%$ of the chicks that failed to hatch could not complete the required rotation on day 35 and therefore suffocated in the egg. Although the rest of the DIS chicks were in approximately the correct position, they failed to hatch. The reasons for this warrants further research, which is also needed to refine the DIS positions before trying to classifying them.

\section{Acknowledgements}

The authors are grateful to the Western Cape Department of Agriculture and Oudtshoorn Research Farm for the use of the resource flock and facility. They thank I. Janse and U. Izaks for opening all the DIS eggs to record the data on the positions of the chicks for this study.

\section{Authors' contributions}

ZB conducted the research during her $\mathrm{PhD}$ studies and was responsible for drafting and submitting the manuscript. SWPC critically analysed and interpreted results. IAM and CRB, together with the SWPC, assisted in the revision of the original and revised manuscript.

\section{Conflict of Interest}

There are no conflicts of interest.

\section{References}

Blood, J.R., Van Schalkwyk, S.J., Cloete, S.W.P. \& Brand, Z., 1998. Embryonic deaths in relation to water loss of artificially incubates ostrich eggs. In: Ratites in a competitive world. F.W. Huchzermeyer (ed). Proc. 2nd Int. Ratite Cong., Oudtshoorn, South Africa. pp. 148-151.

Brand, Z., Cloete, S.W.P., Brown, C.R. \& Malecki, I.A., 2007. Factors related to shell deaths during artificial incubation of ostrich eggs. J. S. Afr. Vet. Assoc. 78, 195-200. 
Brand, Z., Cloete, S.W.P., Brown, C.R. \& Malecki, I.A., 2008. Genetic relationships between water loss and shell deaths in ostrich eggs, assessed as trait of the female. Aust. J. Exp. Agric. 48, 1326-1331.

Brand, Z., Cloete, S.W.P., Brown, C.R. \& Malecki, I.A., 2011. The influence of incubation management on pipping position, hatching ability and survival of ostrich chicks. S. Afr. J. Anim. Sci. 41, 265-274.

Brown, C.R., Peinke, D. \& Loveridge, A., 1996. Mortality in near-term ostrich embryos during artificial incubation. Brit. Poultry Sci. 37, 73-85.

Bunter, K.L., \& Cloete, S.W.P., 2004. Genetic parameters for egg-, chick- and live-weight traits recorded in farmed ostriches (Struthio camelus). Livest. Prod. Sci. 91, 9-22.

Byerly, T.C. \& Olsen, M.W., 1936. Lethality of embryo malpositions in the domestic fowl. Poult. Sci. 15, 158162.

Cloete, S.W.P., Lambrechts, H., Punt, K. \& Brand, Z., 2001. Factors related to high levels of ostrich chick mortality from hatching to 90 days of age in an intensive rearing system. J. S. Afr. Vet. Assoc. 72, 197202.

Deeming, D.C., 1994. When to help ostrich chicks to hatch. The Ostrich News, July 1994. pp. 60-61.

Deeming, D.C., 1995. The hatching sequence of ostrich (Struthio camelus) embryos with notes on development as observed by candling. Brit. Poultry Sci. 36, 67-78.

Deeming, D.C., 1997. Ratite egg incubation. A practical guide. Ratite Conference Books, Buckinghamshire, U.K. pp. 29-50.

Deeming, D.C., Ayres, L. \& Ayres, F.J., 1993. Observations on the first commercial production of ostrich (Struthio camelus) eggs in the UK: Incubation. Vet. Rec. 132, 602-607.

Ipek, A. \& Sahan, U., 2004. Effect of breeder age and breeding season on egg production and incubation in farmed ostriches. Poult. Sci. 45, 643-647.

Ley, D.H., Morris, R.E., Smallwood, J.E. \& Loomis, M.R. 1986. Mortality of chicks and decreased fertility and hatchability of eggs from a captive breeding pair of ostriches. J. Am. Vet. Med. Assoc. 189, 11241126.

Sahan, U., 2003. Near-term embryonic mortalities during artificial incubation of the ostrich egg. Ind. Vet. J. 80, $1002-1005$.

Van Ark, H., 1990. Freq.exe - For the comparison of frequencies. Datametrical Services, Department of Agriculture, Private Bag X116, Pretoria 0001, South Africa.

Van Schalkwyk, S.J., Cloete, S.W.P. \& De Kock, J.A., 1996. Repeatability and phenotypic correlations for body weight and reproduction in commercial ostrich breeding pairs. Br. Poult. Sci. 37, 953-962.

Van Schalkwyk, S.J., Brand, Z., Cloete, S.W.P. \& Blood, J.R., 1998. The influence of different disinfectant protocols on the hatching performance of ostrich chicks. In: F.W. Huchzermeyer (ed). Ratites in a competitive world. Proc. 2nd Int. Ratite Cong., Oudtshoorn, South Africa. pp. 157-159.

Van Schalkwyk, S.J., Brand, Z., Cloete, S.W.P. \& Brown, C.R., 1999. Effect of time of egg collection and preincubation treatment on blastoderm development and embryonic mortality in ostrich embryos. S. Afr. J. Anim. Sci. 29, 154-163. 